\title{
Assessment of training and selected factors on speed and quality of performing different tasks on the endoscopic simulator
}

\author{
Maciej Kasprzyk, Michał Łuczak, Nel Kaczmarek, Jakub Psiuk, Marta Twardowska, Piotr Czarnecki \\ Department of Traumatology, Orthopaedics and Hand Surgery, Poznan University of Medical Science, Poznan, Poland
}

Videosurgery Miniinv

DOI: https://doi.org/10.5114/wiitm.2020.97364

\begin{abstract}
Introduction: One of the most significant challenges nowadays is to educate and predict the predispositions of young surgeons taking into consideration that every ability has its own learning curve.

Aim: To determine the influence of selected factors and examine the shape and the length of the learning curve in performing simple tasks on an endoscopic simulator.

Material and methods: Twenty students took part in 4 training sessions with a one-week break between sessions. They were training 12 minutes and performed three tasks at every session on the endoscopic simulator. To identify whether selected factors influence the time of completing tasks, the participants were asked to fill in questionnaires. All participants also completed the Minnesota Manual Dexterity Test (MMDT) to assess hand-eye coordination.

Results: Our research reveals that regardless of activities performed in free time, the shape of the learning curve was logarithmic. Improvement after the fourth session ranged from 50\% to 75\%. Performing specific activities in the free time did not influence the results achieved on the simulator. No statistically significant correlation between MMDT results and the time to accomplish each task was found.

Conclusions: This study has shown that the length of the learning curve of performing simple tasks is quite short and the shape is logarithmic. It suggests that more complex exercises should be included in the training programme.
\end{abstract}

Key words: learning curve, endoscope, training simulator, surgical resident training.

\section{Introduction}

Endoscopy plays a vital role as a method of diagnosis and treatment in different fields of modern surgery. This is because it is less invasive and usually has a shorter recovery time. For a long time, medical doctors used different types of endoscopy to make a proper diagnosis. The first description of anoscopy and treatment of haemorrhoids is available in the works of Hippocrates [1]. John Macintyre manufactured the first self-illuminated endoscope at Glasgow Royal Infirmary in 1894 or 1895 . Since then, we have made tremendous progress by applying endoscopy in nearly every surgical subspecialty.
One of the elements of evaluating surgical procedures is their safety. To increase patient safety and support surgical education, surgeons should train their surgical skills. They gain experience through fellowship programmes under the supervision of the licensed practitioner. It is widely believed that surgeons should be talented manually and have other predispositions such as resistance to stress and the ability to make quick decisions. One of the most significant challenges nowadays is to educate and predict the preferences of young surgeons taking into consideration that every skill has a learning curve.

Endoscopic simulators could help in perfecting skills before operating on patients.

\section{Address for correspondence}

Jakub Psiuk, Department of Traumatology, Orthopaedics and Hand Surgery, Poznan University of Medical Science, Poznan, Poland,

e-mail: jakubpsiuk@gmail.com 
Although according to Grantcharov [2] preparation for becoming good at technical skills should concern three main components - the cognitive knowledge surrounding the specific medical conditions, instruction enabling abilities that will prepare students by giving them the fundamental elements needed to perform particular procedures and an opportunity to perform a procedure in an environment that is safe and close to real conditions - it could be done outside the clinical setting, reducing the number of errors and increasing the speed of performing the task on patients, which is crucial in times of pressure on speed and maximum efficiency.

\section{Aim}

The aim of the study was to investigate the shape and the length of the learning curve in performing three simple tasks on the endoscopic simulator in relation to selected factors (playing a musical instrument, doing physical activity, playing computer games, time achieved in the Minnesota Manual Dexterity Test).

\section{Material and methods}

To perform this study, the Laparo Aspire endoscopic simulator was used (Photo 1). This trainer is quite simple equipment that needs to be connected to a computer and allows simple tasks to be performed with endoscopic instruments. Also it is rather inexpensive, at about $\$ 400$. It is equipped with several training modules - three of them were used in our research.

A group of 20 students was recruited for the study of the Poznan University of Medical Sciences. Each participant took part in 4 sessions, and each course was composed of two parts: 12 min of self-directed training and completing three tasks. Photo 2 shows tasks that had to be completed in each session.

The initial state on the left and the final state on the right. First row - transferring balls between containers. Second row - change in the configuration of elastic bands. Third row - placing a rubber line through circles.

Tasks that need to be completed on each course are: (1) to move small balls between containers; (2) to change the configuration of elastic bands stretched between sticks; (3) to pass a rubber line through circles.

The time that was spent to complete each task during each session was measured.

To find factors that may influence the participant's performance, all students were asked to fill in two surveys: one at the very beginning of the study, and the second before each session.

In the first survey the following data were collected: age, sex, field of study, year of study, handedness, type of sport that is regularly played (if any), whether he/she plays a musical instrument, amount of time that he/she spends and has spent on playing computer games, chronic disease and whether he/ she has ever used endoscopic equipment.

In the second survey (that was carried out before each session) the following data were collected: level of stress, number of hours that were spent on sleeping last night, drinking coffee or energising drinks, smoking, physical activity in previous $24 \mathrm{~h}$, drinking alcohol in last $24 \mathrm{~h}$, taking drugs in last $24 \mathrm{~h}$.

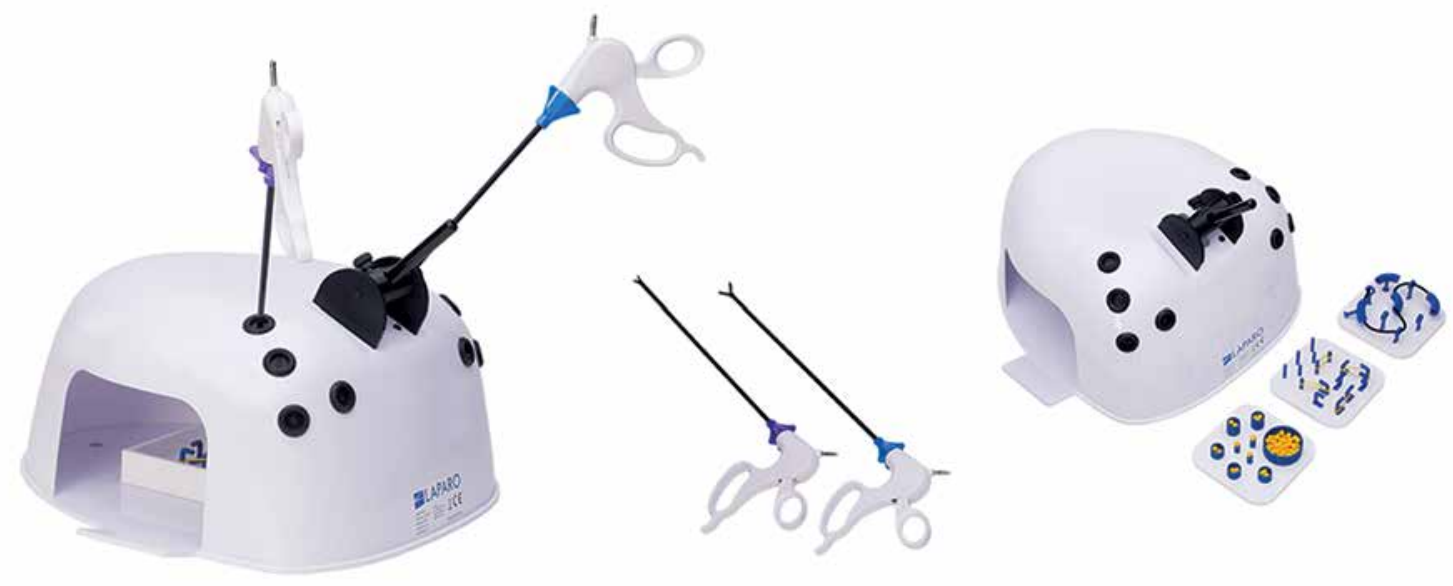

Photo 1. Laparo Aspire endoscopic simulator (image from https://laparo.pl/pl/) 

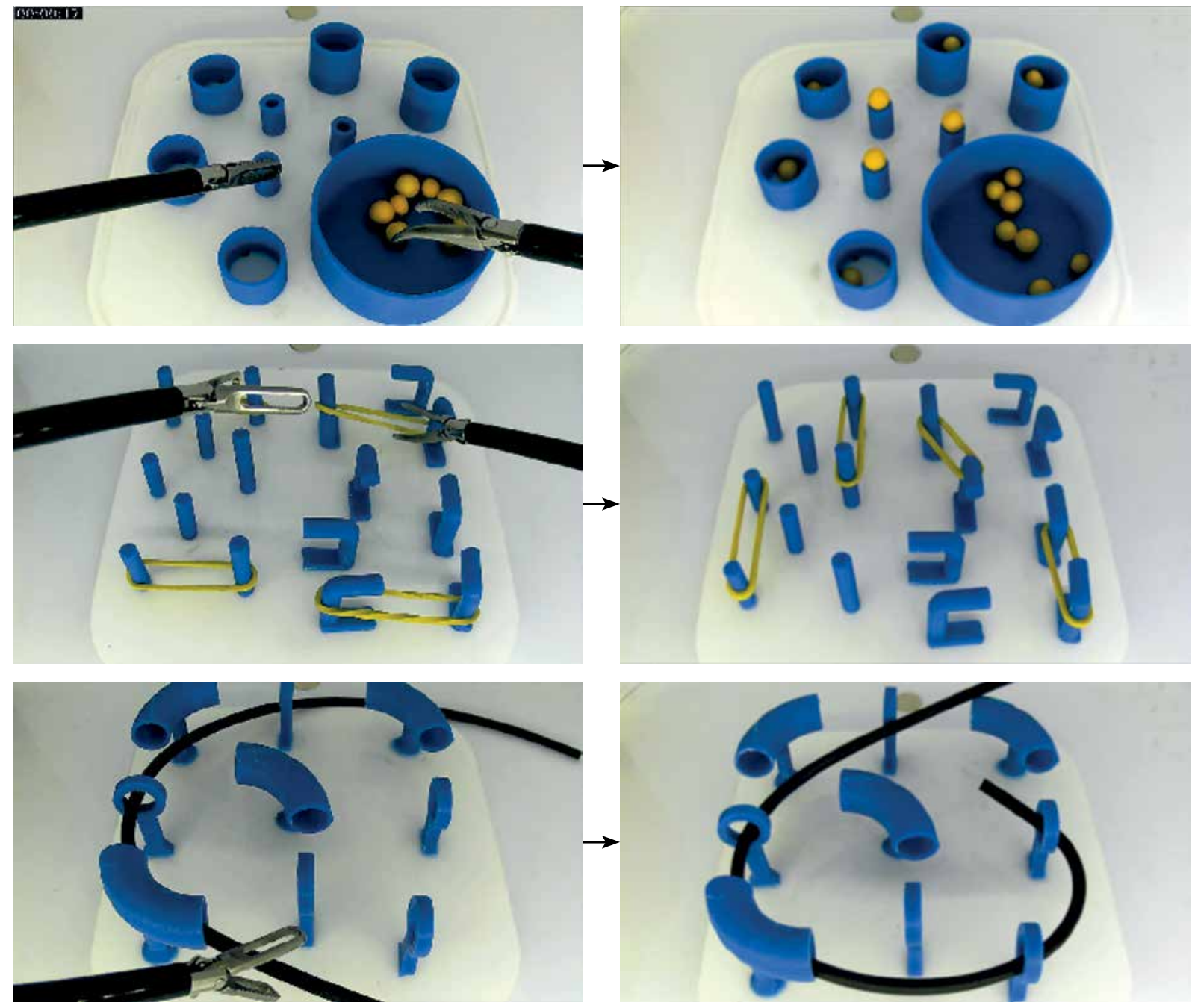

Photo 2. Tasks performed by participants

The Minnesota Manual Dexterity Test (MMDT) is a unified test for rating an ability to move small objects for various distances. It measures the subject's ability of rapid eye-hand coordination and arm-hand dexterity. It was used previously in occupational therapy, and physical therapy, especially when assessing progress after using different treatment methods due to injuries or rehabilitation [3-5].

Participants were asked to perform one of the subtests of this test - the placing test [4]. We measured four trials of the placing test, and we calculated the average time of performing this test.

\section{Statistical analysis}

The statistical analysis was conducted using the Statistica 12 program. The license was free for educational use.

\section{Results}

The mean age of the participants was 22.95 years (range: 20-29 years). Fourteen men and six women took part in our research.

Our research revealed that the mean time of performing each task decreased in every session (when compared to the previous session). All participants were right-handed and studied medicine (Figure 1).

Improvement in time of performing tasks after the fourth session was $75 \%$ for the first task (moving small balls between containers), $50 \%$ for the second task (changing configuration of elastic bands stretched between sticks) and $67 \%$ for the third exercise (passing a rubber line through circles). The Friedman test with the post-hoc test (Dunn Bonferroni test) was performed to determine whether dif- 


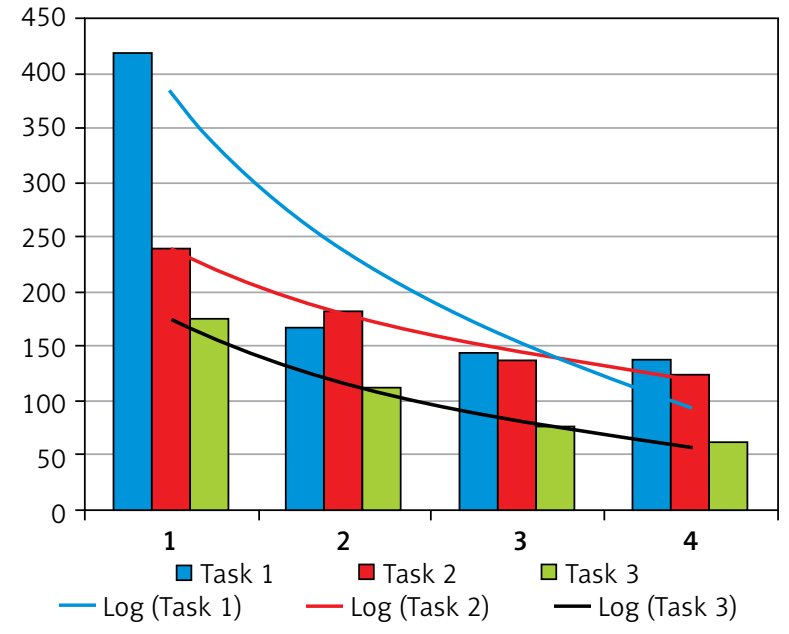

Figure 1. Average time of performing tasks on each training day (in seconds) and logarithmic learning curve fitting

ferences between times of completing each task in each session were statistically significant. For each task, a statistically significant difference occurred between different sessions (Table I).

Ramsay et al. have shown that the learning curve can be approximated in the most accurate way by a logarithmic curve. Based on that calculated logarithmic curve, coefficients for each task were calculated.

According to that, it seems that each task has its own level of difficulty. A significant correlation between the learning curve of transferring rubber bands (task 2) and threading (task 3) was documented, thus suggesting similar complexity of these tasks.

Figure 1 shows approximated learning curves of tasks $1-3$. The mean time of performing MMDT was 57 s (range: 44-75 s).

To assess whether there is a correlation between the MMDT result and the time to accomplish each task, Spearman's rank correlation coefficient was calculated. No statistically significant correlation was found. Also, Spearman's rank correlation coefficient for the comparison between tasks was carried out, and a significant correlation $(p<0.05)$ was found.

Comparison of participants who used to take part in specific activities in their free time with those who did not revealed a significant difference in results achieved on the simulator. Playing music, playing computer games and doing physical activity were assessed (Table II).

\section{Discussion}

The goal of the study was to determine the shape of the learning curve of laparoscopic work and evaluate whether chosen factors affect the speed of task execution.

After reviewing the available medical literature, we noted that several similar studies focus on the use of various simulation methods and the resulting improvement in abilities, not only in orthopaedics or surgery but in medicine in general. Training and simulation are seen today as an essential element in medical education and are increasingly appearing in the teaching of students and residents, ensuring more excellent patient safety [6]. It is also necessary to emphasise a higher value of the practical training over the observation itself [7]. However, as Panek et al. reported, not only manual skills influence the learning curve of an individual but also their experience with regard to the entire therapeutic process, including choice of the optimal surgical method [8].

It has been proven that much more expensive virtual reality simulators bring equal benefits to those in training, compared to box trainers, to which the Laparo Aspire belongs [9-11]. Therefore, one can confirm the benefits of the availability of training on box trainers, which one can even take home. However, even though they can effectively support the laparoscopic training, personal possession of

Table I. Results of Friedman test with post-hoc test (Dunn Bonferroni) for each task (yes - significant difference, no - no significant difference)

\begin{tabular}{|lccccccccc|}
\hline $\begin{array}{l}\text { Session } \\
\text { number }\end{array}$ & \multicolumn{1}{c}{ Exercise 1 } & \multicolumn{3}{c}{ Exercise 2 } & \multicolumn{3}{c|}{ Exercise 3 } \\
\hline 2 & 1 & 2 & 3 & 1 & 2 & 3 & 1 & 2 & 3 \\
\hline 3 & Yes & & & No & & No & \\
\hline 4 & Yes & No & & Yes & Yes & Yes & No \\
\hline
\end{tabular}


a portable simulator does not result in voluntary long-term practice [12].

In the studies comparing also the mental training, which turns out to be very important in the case of additional stress factors appearing during an operation $[13,14]$, one can note the decisive advantage of training on box simulators and virtual training simulators over mental training [15] in the matter of laparoscopic performance, which also confirms the validity of practical training on simulators. There are also studies available that support the superiority of training on box trainers over virtual reality trainers [16].

To assess the length and shape of the learning curve of laparoscopic work and factors affecting the speed of task execution, 20 medical students completed questionnaires and performed training on the endoscopic simulator. The findings indicate a logarithmic learning curve with a marked improvement in results after all training and research sessions. No error frequency was recorded as it was assumed that the error would directly affect the time necessary to complete the task. The decrease in path length, which can be used for assessment in curricula for laparoscopic cholecystectomy $[17,18]$ and rating in surgical skills [19] is seen by some as a somewhat manual skill, developed with task repetition. However, error frequency is a crucial component in the clinical marker, and the indicator of the quality of the operation [20] was left unrecorded. It has been shown that a decrease in error frequency number is possible to achieve in structured coaching [18], which was lacking in our study.

Cumpanas et al. [21] examined how sleep deprivation influenced performance on the robotic virtual reality simulator. In their research sleep deprivation does not influence the time of performance on easier tasks but influences time of performing more difficult tasks.

During training sessions, we noted that the type of exercise performed in the current study had a positive effect on the students' abilities. The Laparo Aspire simulator proved to be an excellent learning method, mainly for simple tasks. What is more, for students who have never had a chance to deal with laparoscopic work, such training was an introduction to a two-dimensional vision. It is undoubtedly one of the significant difficulties faced by amateurs of laparoscopic surgery.

During the university classes, medical students have practically no contact with the laparoscope,
Table II. Number of participants doing specific activities in their free time and results of comparing groups

\begin{tabular}{|lccc|}
\hline Parameter & Yes & No & $P$-value \\
\hline Musical instruments & 5 & 15 & 0.735423 \\
\hline Physical activity & 15 & 5 & 0.497291 \\
\hline Computer games & 11 & 9 & 0.823756 \\
\hline
\end{tabular}

apart from observing the procedures carried out by older, experienced surgeons. Medical students and young adepts of orthopaedics and surgery should have constant contact with laparoscopic trainers [21-23].

In contradiction to Buckley CE's findings, which state that high aptitude is directly related to earlier completion of the learning curve [24], our studies did not show a significant correlation between the results in the MMDT test and the laparoscopic work.

The weakness of our study might be an insufficient number of surveyed students, which could be a reason for limited and inadequate trends for initial aptitude and the length of the learning curve. Despite the size of our group of participants, our findings that laparoscopic training improves performance correlate with those of others [25, 26]. We should also consider introducing more complex tasks to lengthen the learning curve.

To further advance our study, we would also like to compare the results that the students achieved with the results achieved by the experienced surgeons who work laparoscopically daily, to confirm the trends observed by other researchers [27].

One may suspect that, as in some studies [28], training on the box trainer can produce similar results compared to traditional methods, where experienced surgeons educate trainees through the process of graduated responsibility. On the other hand, we think that, based on the learning curve in our research, results of a randomised controlled trial that show how laparoscopic surgical skills are significantly improved by use of a portable laparoscopic simulator [29] and other studies proving that warmup sessions before operations cause improvement of performance $[30,31]$ training on the box trainers and/or laparoscopic simulators may be widely and effectively used in many different aspects. As other researchers discovered, many attempts are required in different surgery cases to stabilize the learning 
curve [32]. That is why targeted training sessions are required to obtain sufficient proficiency .

\section{Conclusions}

The assessment made in our study suggests that regular training on an endoscopic simulator significantly decreases the time needed to perform different tasks (both in general and compared to the previous session). We did not find a significant correlation between initial aptitude and the time necessary to complete given tasks. What is more, we should consider comparing students with experienced surgeons.

\section{Conflict of interest}

The authors declare no conflict of interest.

\section{References}

1. Jani PG. Management of haemorrhoids - a personal local experience. East Cent Afr J Surg 2005; 10: 24-28.

2. Grantcharov TP, Reznick RK. Teaching procedural skills. BM 2008; 336: 1129-31.

3. Tesio L, Simone A, Zebellin G, et al. Bimanual dexterity assessment: validation of a revised form of the turning subtest from the Minnesota Dexterity Test. Int J Rehabil Res 2016; 39: 57-62.

4. Lourenção MI, Battistella LR, Martins LC, et al. Analysis of the results of functional electrical stimulation on hemiplegic patients' upper extremities using the Minnesota manual dexterity test. Int J Rehabil Res 2005; 28: 25-31.

5. Hutzler Y, Lamela Rodríguez B, Mendoza Laiz N, et al. The effects of an exercise training program on hand and wrist strength, and function, and activities of daily living, in adults with severe cerebral palsy. Res Dev Disabil 2013; 34: 4343-54.

6. Ramsay CR, Grant AM, Wallace SA, et al. Statistical assessment of the learning curves of health technologies. Health Technol Assess 2001; 5: 1-79.

7. Schmitt F, Mariani A, Eyssartier E, et al. Learning laparoscopic skills: observation or practice? J Laparoendosc Adv Surg Tech A 2018; 28: 89-94.

8. Panek W, Szmer J, Kuijper CF, et al. Learning curve or experience-related outcome: what really matters in paediatric laparoscopic pyeloplasty. Videosurgery Miniinv 2020; 15: 377-81.

9. Diesen DL, Erhunmwunsee L, Bennett KM et al. Effectiveness of laparoscopic computer simulator versus usage of box trainer for endoscopic surgery training of novices. J Surg Educ 2011; 68: 282-9.

10. Yiasemidou M, Siqueira J, Tomlinson J, et al. "Take-home" box trainers are an effective alternative to virtual reality simulators. J Surg Res 2017; 213: 69-75.

11. Khan MW, Lin D, Marlow N, et al. Laparoscopic skills maintenance: a randomized trial of virtual reality and box trainer simulators. J Surg Educ 2014; 71: 79-84.

12. Zapf MA, Ujiki MB. Surgical resident evaluations of portable laparoscopic box trainers incorporated into a simulation-based minimally invasive surgery curriculum. Surg Innov 2015; 22: 83-7.

13. Anton NE, Beane J, Yurco AM, et al. Mental skills training effectively minimizes operative performance deterioration under stressful conditions: results of a randomized controlled study. Am J Surg 2018; 215: 214-21.

14. Stefanidis D, Anton NE, Howley LD, et al. Effectiveness of a comprehensive mental skills curriculum in enhancing surgical performance: results of a randomized controlled trial. Am J Surg 2017; 213: 318-24.

15. Mulla M, Sharma D, Moghul M, et al. Learning basic laparoscopic skills: a randomized controlled study comparing box trainer, virtual reality simulator, and mental training. J Surg Educ 2012; 69: 190-5.

16. Brinkmann C, Fritz M, Pankratius U, et al. Box- or virtual-reality trainer: which tool results in better transfer of laparoscopic basic skills? A prospective randomized trial. J Surg Educ 2017; 74: 724-35.

17. Aggarwal R, Crochet P, Dias A, et al. Development of a virtual reality training curriculum for laparoscopic cholecystectomy. Br J Surg 2009; 96: 1086-93.

18. Zhang A, Hünerbein M, Dai Y, et al. Construct validity testing of a laparoscopic surgery simulator (Lap Mentor): evaluation of surgical skill with a virtual laparoscopic training simulator. Surg Endosc 2008; 22: 1440-4.

19. Pellen M, Horgan L, Roger Barton J, et al. Lapa- roscopic surgical skills assessment: can simulators replace experts? World I Surg 2009; 33: 440-7.

20. Lonergan PE, Mulsow J, Tanner WA, et al. Analysing the operative experience of basic surgical trainees in Ireland using a web-based logbook. BMC Med Educ 2011; 11: 70.

21. Cumpanas AA, Ferician O, Lațcu S, et al. Does sleep deprivation alter virtual reality-based robotic surgical skills? Videosurgery Miniinv 2020; 15: 97-105.

22. van Empel PJ, Verdam MG, Strypet M, et al. Voluntary autonomous simulator based training in minimally invasive surgery, residents' compliance and reflection. J Surg Educ 2012; 69: 564-70.

23. Teitelbaum EN, Soper NJ, Santos BF, et al. A simulator-based resident curriculum for laparoscopic common bile duct exploration. Surgery 2014; 156: 880-93.

24. Buckley CE, Kavanagh DO, Nugent E, et al. The impact of aptitude on the learning curve for laparoscopic suturing. Am J Surg 2014; 207: 263-70.

25. Howells NR, Gill HS, Carr AJ, et al. Transferring simulated arthroscopic skills to the operating theatre: a randomised blinded study. J Bone Joint Surg Br 2008; 90: 494-9.

26. Frank RM, Erickson B, Frank JM, et al. Utility of modern arthroscopic simulator training models. Arthroscopy 2014; 30: 121-33.

27. Tashiro Y, Miura H, Nakanishi Y, et al. Evaluation of skills in arthroscopic training based on trajectory and force data. Clin Orthop Relat Res 2009; 467: 546-52.

28. Marlow N, Altree M, Babidge W, at al. Laparoscopic skills acquisition: a study of simulation and traditional training. ANZ J Surg 2014; 84: 976-80.

29. Johnston TJ, Tang B, Alijani A, et al. Laparoscopic surgical skills are significantly improved by the use of a portable laparoscopic 
simulator: results of a randomized controlled trial. World I Surg 2013; 37: 957-64

30. Kroft J, Ordon M, Arthur R, et al. Does surgical 'warming up' improve laparoscopic simulator performance? Simul Healthc 2012; 7: 339-42.

31. Chen CC, Green IC, Colbert-Getz IM, et al. Warm-up on a simulator improves residents' performance in laparoscopic surgery: a randomized trial. Int Urogynecol J 2013; 24: 1615-22.

32. Rubinkiewicz M, Truszkiewicz K, Wysocki M, et al. Evaluation of the learning curve of transanal total mesorectal excision: single-centre experience. Videosurgery Miniinv 2020; 15: 36-42.

Received: 7.04.2020, accepted: 9.06.2020. 